\title{
A NOVEL 4-SENSOR SYSTEM FOR FALL DETECTION AND ABNORMAL MEDICAL SITUATIONS IDENTIFICATION
}

\author{
Gairat Kuttumuradov, Arslan Mukhamatnurov, Nurmukhamed Tursumbek and Laurent Billonnet \\ University of Limoges, Limoges, France
}

\begin{abstract}
Many organizations and industries already provide turnkey solutions for the detection of falls, but many of these systems are too expensive. Most of people like living independently at home. However, many people are not able to afford expensive systems to detect or prevent falls. Many people are not even thinking about using it. Some activities in our daily life can lead to accidents, such as falls. The fall are defined as unintentionally coming to rest on the ground, floor or other lower levels. Falls can lead people to fatal conditions, even death. We propose here a four-sensor concept to improve the efficiency of the system and reduce its global cost. A basic concept of fall detection system using three inclinometers and a heartbeat sensor based on Smartphone is presented. Inclinometers and heartbeat sensors are worn in different part of human body to detect fall more accurately. Automatic alert call will be sent to family members or caregivers when a fatal condition is identified. Our system is quite innovative since it can distinguish several situations critical or not by combining data coming from the four sensors. Thus, the system can also monitor daily activities to improve daily life of the users.
\end{abstract}

\section{KEYWORDS}

Inclinometer, Heartbeat Sensor, Falls, Abnormal Situation

\section{INTRODUCTION}

The fall is an accident that threatens health, especially for older people. Fall detection has thus become an important research field. Every year around the world, about 646,000 people die from falls, of which more than $80 \%$ are in low and middle-income countries. Although not fatal, an estimated 37.3 million falls become serious enough to demand medical care every year. Very often, the high mortality rate among older people is due to a fall and falls are the second leading cause of death from accidental or unintentional injuries worldwide. This problem affects older people aged 65 and above. The reason is the decrease in strength and stability of the human body. Preventing a fall is also a difficult problem. At least, a reliable detection of falls can prevent the worst consequences (severe physical or psychological impacts and in some cases death).

Nowadays, different types of fall detection system based on cameras exist. But most of people worry about the privacy of information of themselves so that no one wants to stay under camera in his daily living. However, this is not the main reason to doubt to this fall detection system. An omni-camera is a camera with 360 -degree field of view. Successful rate of this system is approximately $80 \%$, which means that in 1 case out of 5, the fall is not detected and can lead to user's death. The causes of falls are often the result of complex interactions between many factors. World Health Organization has identified more than 400 factors that contribute to a fall. In addition, falls of elderly result from the effect of additive factors.

Thus, screening the factors may help reduce the risk of falling. In fact, the risk of falling and the number of factors are linearly correlated. Risk factors are often classified in three dimensions. There are factors related to the behaviour, other factors linked to the health status of the elderly and finally factors linked to the environment (extrinsic factors). The first two dimensions can be merged together. They score the factors associated to the person (intrinsic factors). Falls happen because of the interaction among these dimensions as illustrated in figure 1. 


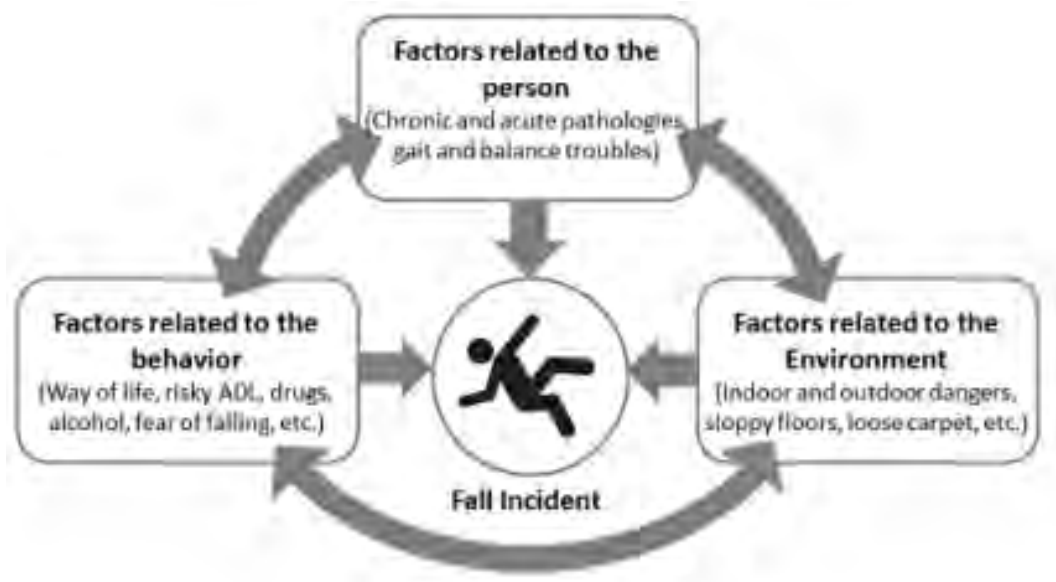

Figure 1. Three dimensions of fall risk factors

Because people usually want to feel safe in their house, indoor system can be more relevant. However, probability of the fall increases outside. In this case, wearable devices have to be integrated in the global solution. It means that wearable device has to be useful and comfortable to wear. Luckily, the development of wearable devices and mobile Internet gives chance to deal with the problem. Wearable devices based on health care have seeped in our daily life and changed our lifestyle a lot with great benefit.

\section{DATA TRANSMISSION}

Our system is based upon a 4-step process: data collection, data transmission, data processing and fall alert.

Modern Smartphones have excellent information processing via to their advanced processors. By collecting data from the inclinometers and sending it to the Smartphone, the Smartphone's application is able to analyse the data from the inclinometers and extract decision parameters. To accurately determine the fall, the system reads data coming from the inclinometers every second. Otherwise, we should expect a delay of several seconds, which will adversely affect the quality of this system. After processing of information, the application recognizes the user's position and situation and in case of alert criterion identification (i.e., the parameters recognizing a True positive situation of fall) the system sends a fall alert in real-time.

In addition, the heartbeat sensor gives more accuracy and relevance. Its focus is the determination of abnormal medical situations in the pre fall phase. This is very important since it proves that our system can also prevent from falls, or, at least, identify the high probability of fall before it occurs.

The connection between the sensors will be established by Bluetooth low-energy, which allows the sensors to transmit information at a frequency of $2.4 \mathrm{GHz}$ with low power consumption. The packet transfer rate in this case is about $1 \mathrm{Mbit} / \mathrm{s}$. Consuming less power; Bluetooth low-energy technology will offer long-lasting connectivity and connect small devices such as sensors. It should also be noted that the standard Bluetooth network supports one master and maximum of seven slaves. In our case, we use four sensors as slaves driven by the smartphone that is the main device (i.e. the master).

\section{MONITORING ACTIVITIES AND HEALTH}

As mentioned above, our system is, strictly speaking, unable to prevent a fall, but thanks to information collection in real time, it is able to almost predict a fall (through the use of the heartbeat sensor) and at minimum to determine a person's position in real time.

To do this, three inclinometers are placed on different parts of the human body and a heartbeat sensor is worn through a connected smartwatch, on a soft skin area (with a cluster of blood vessels) for greater sensitivity of the sensor (Figure 2). 


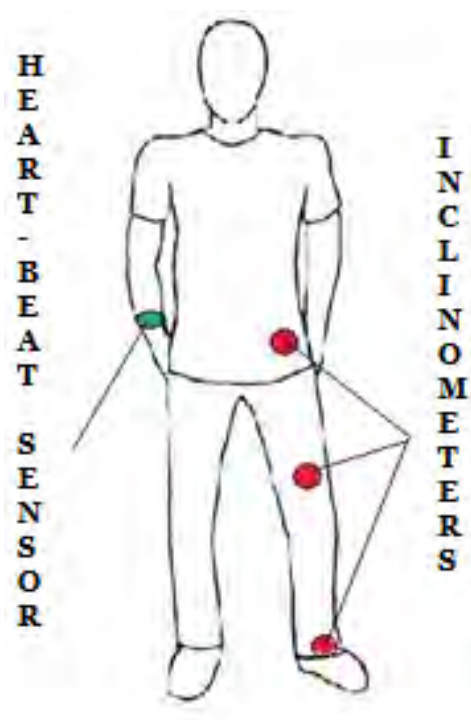

Figure 2. Four-sensor system

The location of the sensors plays a significant role in this system. Moreover, in order to the sensors to be mounted firmly on the human body, the attachment to patches is implied. We cannot consider attaching the sensors to clothes, since in this case the sensors will not be on a good mount and will start to hang out, which will lead to a disruption of the system and to a possible wrong analysis of the data.

Even despite the cheapness of this system, the convenience of the size of the sensors and its effectiveness, it is not suitable for all people. The main problem is people with cognitive disorders, leading to impaired mental activity and memory loss. It means that people entering this category will periodically forget to wear these sensors to ensure their safety.

An inclinometer is a device designed to measure the angle of inclination of various objects relative to the gravitational field of the Earth. They are used in cameras, aircraft flight controls, automobile security systems, switches and are used for platform levelling, boom angle indication, and in other applications requiring measurement of tilt. One of the inclinometers is installed on the thigh to calibrate the user's torso in order to understand at what angle the upper part of the human body is located. The second is on the upper leg. The third inclinometer is placed on the lower leg. Then, three inclinometers are enough to understand in what position the user's body is.

Table 1 shows the user's basic positions such as standing, sitting and lying positions. When a user moves the angle of inclinometers changes with time. The variety speed of angle inclinations depends on the movements of the user. By receiving data from the inclinometers in real-time, the system recognizes the position and variety speed of angle inclination, thereby understanding in which of the positions the user is. In addition, using time as a parameter, the system is able to monitor in real-time the user's activity. This analysis thus enables not only to evaluate the activity itself (number of steps, walking pace, calories burned, etc.) but also to show a drift in the wandering of the person and then to predict the probability of a fall.

The three inclinometers enables to efficiently identify main positions in almost all classical daily activities. In particular, stand up positions, sitting and lying position can effectively tracks position of the body. In correlation with the heartbeat sensor, the system is able to link the position and the health status of the user. For example, a user can be sitting with a normal heartbeat pulse. This means that he is normally sitting.

But if the inclinometers identify a sitting position while the heartbeat pulse is too high or too low, this corresponds to an critical situation. The efficiency is the same in the lying position. If the user is lying with a normal heartbeat pulse, then he is just resting or sleeping. In the same position, if the heartbeat is too high or too low, then a critical situation is also identified.

Table 1. Usual positions of human body for daily activities

\begin{tabular}{llll}
\hline Position & Sensor 1 angle & Sensor 2 angle & Sensor 3 angle \\
\hline Standing up & $90^{\circ}$ & $90^{\circ}$ & $90^{\circ}$ \\
Sitting & $90^{\circ}$ & $0^{\circ}$ & $90^{\circ}$ \\
Lying & $0^{\circ}$ & $0^{\circ}$ & 0 \\
\hline
\end{tabular}


Pulse measurement using optical plethysmography is the most common way to measure the pulse and is now implemented in sports watches, trackers, mobile phones. Light entering the bloodstream will be quite predictably scattered when the blood flow rate changes (for example, an increase in cardiac output). The optical technology measures the pulse using LEDs that evaluate blood flow to the wrist. This means that you can measure your pulse without using a chest strap. In practice, the optical sensor on the back of the watch emits light on the wrist using LEDs, and measures the amount of light scattered by the bloodstream.

At final step, user's data is automatically read and transmitted to the smartphone which application processes the data for output parameters. In the case of high probability of an abnormal situation, the application can also send a request to a heartbeat pulse reading to the watch. At this point, the user can ask for help or reject the request if everything is all right. If the user does not validate in any manner after 15 seconds, the system automatically calls for help.

Using time as a parameter, our system is also able to monitor the user's activity. In particular, for fragile people, it is able to measure a drift in the rhythm of daily life processes such as walk. But it is also able to determine the duration of the activities and to quantify the level of activities. That can also be useful for people doing sports to measure the performance itself but also to ensure the activity is done safely.

Using the same approach, the system can be used with benefit for people in reeducation or rehabilitation (for example after an accident when they are back home). The system is then used as a coach to validate the efficiency of the physical exercises and activities of the user in terms of intensity and rhythm.

That shows that our system is then much more than a fall detection system but can also be used with great flexibility for a lot of health and well-being (even well-ageing) applications at low cost.

\section{CONCLUSION}

Nowadays, the gerontechnology sphere is actively developing because we are facing the problem of active aging of the population. This paper focuses on the problem of falling. The fall is one of the main causes of unintentional injuries for the elderly. After analyzing the disadvantages of the existing solutions, we propose a system capable of compensating for this range of deficiencies.

This paper considers problems of falls and presents the fall detection system based on three inclinometers, pulse meter and smart watch, designed for the elderly. The system provides automatic alerts in case of fall. In comparison of other existing solutions, our concept provides several advantages, such as possibility of monitoring outdoor, ability to determine the abnormal medical records of the user through his heartbeat, determination of user location (in critical situations), comfort of use, ethical design.

The future possibility of this technology relies in the introduction of artificial intelligence in the system. Artificial intelligence will significantly improve the performance of our system and will allow not only to identify the fall, but also to help in improving the user's physical activity based on statistics compiled.

\section{REFERENCES}

Chen, K. et al., 2016, A System of Fall Detection Using a Wearable Device Based on Bluetooth Communication. Proceedings of the 13th IEEE International Conference on Solid-State and Integrated Circuit Technology (ICSICT). Hangzou, China, p. 1.

Florent Lachal, https://prezi.com/7rk3a2uehhel/fall-sensors/

Li, C. et al., 2018, Novel sensor-fusion system concepts for indoor and outdoor fall detection. Proceedings of the 1st International Conference on Human System Engineering and Design (IHSED 2018). Reims, France.

Matti Siekkinen, Markus Hiienkari, Jukka K. Nurminen and Johanna Nieminen Dept. of Computer Science and Engineering and Nokia Research Center, Finland, How low energy is Bluetooth low energy? Comparative measurements with ZigBee/802.15.4

Rakhman, A. Z., 2014. Fall Detection System Using Accelerometer and Gyroscope Based on Smartphone. Proceedings of the $1^{\text {st }}$ International Conference on Infonnation Technology, Computer and Electrical Engineering, Semarang, Indonesia, pp. 99-100.

World Health Organization, http://www.who.int/news-room/fact-sheets/detail/falls, 2018.

https://www.elprocus.com/heartbeat-sensor-working-application/ 